www.nature.com/pj

\title{
Effects of melt annealing on the miscibility and crystallization of poly(butylene succinate)/ poly(ethylene succinate) blends
}

\author{
Toshiyuki Kataoka ${ }^{1}$, Kohji Hiramoto ${ }^{1}$, Hideo Kurihara ${ }^{2}$ and Takayuki Ikehara ${ }^{1}$
}

We discuss the enhancement in miscibility of crystalline/crystalline blends of poly(butylene succinate) and poly(ethylene succinate) (PBS/PES) by simple melt annealing, their crystallization process and the formation of characteristic phaseseparated structure. The immiscible PBS/PES blends changed into miscible blends when annealed at $150^{\circ} \mathrm{C}$. Gel permeation chromatography and differential scanning calorimetry measurements suggested that the annealing process caused transesterification between the PBS and PES, resulting in the formation of PBS-PES copolyesters. The unannealed blends exhibited phase-separated melts with constituents that crystallized separately. A PBS-rich layer was formed between the PES-rich phase and the glass substrate from the phase-separated melts of the unannealed blends. In contrast, the miscible annealed blends crystallized by two distinct processes. The first process, successive crystallization, involved PBS spherulite formation in the homogeneous melt and PES spherulite nucleation inside the PBS spherulites. The second process involved the simultaneous nucleation of both components from the melt and penetrating growth of the PES spherulites into the PBS spherulites.

Polymer Journal (2014) 46, 405-411; doi:10.1038/pj.2014.11; published online 19 March 2014

Keywords: crystallization; phase behavior; polyester; polymer blend; transesterification

\section{INTRODUCTION}

Polymer blends have been investigated extensively for fundamental research and practical applications. ${ }^{1-17}$ Miscibility and crystalline morphology largely influence the physical properties of polymer blends. In miscible crystalline/crystalline blends, for instance, the difference in melting temperature $\left(T_{\mathrm{m}}\right)$ between the two constituents affects their crystallization behavior. ${ }^{5,7-17}$ If the $T_{\mathrm{m}}$ difference is smaller than $\sim 30 \mathrm{~K}$, the two components crystallize simultaneously from the melts and often form interpenetrating spherulites, where the spherulites of one component continue to grow inside those of the other component. ${ }^{5,7-9}$ For larger $T_{\mathrm{m}}$ differences, however, the higher$T_{\mathrm{m}}$ component crystallizes first, with the lower $T_{\mathrm{m}}$ component crystallizing successively inside the spherulites of the higher $T_{\mathrm{m}}$ component. ${ }^{14,15}$

Most polymer pairs have poor miscibility because they usually have small entropies of mixing. For this reason, substantial effort has been devoted to finding miscible polymer pairs and compatibilizing essentially immiscible polymers. ${ }^{18-30}$

Transesterification is one method commonly used to compatibilize polyester blends. ${ }^{19-30}$ When polyester blends are annealed in a melt, the polyesters convert to block copolyesters by exchanging their main chains. ${ }^{26,27}$ Copolyesters with a larger number of blocks and shorter block lengths form as the interchange reaction repeatedly occurs. Studies on immiscible blends of polyester/polycarbonate have revealed that the copolymers produced through transesterification stay mostly at the interfaces between the phase-separated domains and that the interfacial thickness increases with annealing time. ${ }^{31}$ Although several reports have described the influence of transesterification on crystallization kinetics and morphology, ${ }^{28,32}$ to our knowledge the relationship between transesterification and the formation of interpenetrating spherulites in such blends has not been investigated.

Poly(butylene succinate) (PBS) is a biodegradable aliphatic polyester with good mechanical properties ${ }^{32-39}$ that can be produced from biomass chemicals. ${ }^{32,39}$ Poly(ethylene succinate) (PES) is also a biodegradable polyester with a chemical structure similar to PBS. ${ }^{34-36,40,41}$ PBS/PES blends are typically either immiscible or only partially miscible. ${ }^{3,4,42}$ In our previous work, ${ }^{42}$ miscible PBS/PES blends were obtained by adding poly(ethylene oxide), which is miscible with both PBS and PES. The copolymerization has also been examined to compatibilize PBS and PES, ${ }^{43-48}$ although this process is technically complex $^{43,44}$ and results in a significant decrease in the crystallinity. ${ }^{45}$

Here, we report the crystallization process and morphology of miscible blends of PBS and PES obtained by transesterification through melt annealing. The effects of annealing on the phase

${ }^{1}$ Department of Material and Life Chemistry, Faculty of Engineering, Kanagawa University, Kanagawa, Japan and ${ }^{2}$ Polyolefin Technology Center, Japan Polychem Corporation, Yokkaichi, Japan

Correspondence: Dr T Kataoka, Department of Material and Life Chemistry, Kanagawa University, 3-27-1, Rokkakubashi, Yokohama, Kanagawa 221-8686, Japan.

E-mail: tkataoka@kanagawa-u.ac.jp

Received 20 November 2013; revised 6 January 2014; accepted 14 January 2014; published online 19 March 2014 
behavior and the crystallization are discussed. We obtained fully miscible and highly crystalline PBS/PES blends with mild annealing conditions (annealing temperature $\sim 150{ }^{\circ} \mathrm{C}$ ). Moreover, the immiscible melt formed layered structures in which the copolymers produced through transesterification have stabilized structures.

\section{EXPERIMENTAL PROCEDURE}

$\operatorname{PBS}\left(M_{\mathrm{w}}=83000, T_{\mathrm{m}}=120^{\circ} \mathrm{C}\right)$ and $\operatorname{PES}\left(M_{\mathrm{w}}=10000, T_{\mathrm{m}}=108^{\circ} \mathrm{C}\right)$ were purchased from Sigma-Aldrich Corporation (St Louis, MO, USA) and Scientific Polymer Products, Inc. (Ontario, NY, USA), respectively. The PBS/ PES blends were prepared by a solution-casting method. The two polymers were dissolved together in chloroform. The solvent was allowed to evaporate in Petri dishes overnight, before drying in vacuo at $30{ }^{\circ} \mathrm{C}$ for 2 days. The blends were then annealed at $150{ }^{\circ} \mathrm{C}$ under $\mathrm{N}_{2}$ gas flow to obtain the annealed blends. As an example of our naming convention, the annealed blend 6/4-30m refers to a weight ratio of PBS/PES and an annealing time of $6 / 4$ and $30 \mathrm{~min}$, respectively.

The phase behavior and the crystallization process were observed using phase contrast (BX51, Olympus, Tokyo, Japan) and polarized optical (Olympus, BH-2) microscopes equipped with temperature controllers (LK-600PM, Linkam, Surrey, UK) and CCD cameras (CoolSnap 5.0, Roper Scientific, Tucson, AZ, USA). A sensitive tint plate with a retardation of $530 \mathrm{~nm}$ was used for polarized optical microscopy. A film with a thickness of $\sim 10 \mu \mathrm{m}$ was placed between the two optical glasses. For observation of spherulitic crystal growth, the film was melted above the $T_{\mathrm{m}}$ of $\mathrm{PBS}$ to $130{ }^{\circ} \mathrm{C}$ and then immediately cooled to the crystallization temperature $\left(T_{\mathrm{c}}\right)$ to minimize the effect of transesterification.

To determine the temperature of phase separation, the blend was mounted on a phase contrast microscope. A phase-separated melt was first heated unti it became a homogeneous melt at $\sim 180^{\circ} \mathrm{C}$, and then immediately cooled until the melt phase separated. The reproducibility was carefully checked by replacing the film every time to avoid transesterification at high temperatures.

Gel permeation chromatography (GPC) was performed on an LC-908 system (Japan Analytical Industry Co. Ltd., Tokyo, Japan) using chloroform as an eluent at a flow rate of $3.5 \mathrm{ml} \mathrm{min}^{-1}$ with refractive index detection. A DSC6200 (Seiko instruments Inc., Chiba, Japan) was used for differential scanning calorimetry (DSC). Approximately $5 \mathrm{mg}$ of the sample was sealed in an aluminum pan, melted at $130^{\circ} \mathrm{C}$ and then quenched in liquid $\mathrm{N}_{2}$ prior to the measurement Thermograms were recorded at a heating rate of $10^{\circ} \mathrm{C} \mathrm{min}-1$.

\section{RESULTS AND DISCUSSION}

\section{Phase behavior}

Figures $1 \mathrm{a}$ and $\mathrm{b}$ shows micrographs of the $4 / 6$ unannealed PBS/PES blend. The blend was homogeneous at $180^{\circ} \mathrm{C}$ and phase-separated at $130^{\circ} \mathrm{C}$, indicating that PBS/PES binary blends have a phase diagram with an upper critical solution temperature. The $6 / 4,5 / 5$ and $2 / 8$ unannealed blends of PBS/PES also showed similar phase behavior, whereas the $8 / 2$ blend did not show phase separation above $130{ }^{\circ} \mathrm{C}$. However, the 4/6 PBS/PES blend annealed for $60 \mathrm{~min}(4 / 6-60 \mathrm{~m})$ was a homogeneous melt at $130{ }^{\circ} \mathrm{C}$ (Figure 1c). This clearly indicates that annealing at the melt state is an efficient way to enhance the miscibility between PBS and PES.

We determined the phase separation temperatures by phase contrast microscopy. The phase diagram in Figure 2 shows that the blends are partially miscible at $130^{\circ} \mathrm{C}$. The phase separation temperatures shifted lower as the annealing time at $150{ }^{\circ} \mathrm{C}$ was prolonged. The blends that were annealed for more than $60 \mathrm{~min}$ did not show phase separation at any of the weight ratios.

The miscibility was also confirmed by investigating the glass transition temperature $\left(T_{\mathrm{g}}\right)$ by DSC. Figure 3 shows the DSC thermograms for neat PBS, neat PES, and the blends that were annealed for $0-60 \mathrm{~min}$. Figure 4 shows enlarged curves for the neat polymers and the $4 / 6$ blends around the $T_{\mathrm{g}}$. The $4 / 6-0 \mathrm{~m}$ blend exhibited two $T_{\mathrm{g}} \mathrm{s}$ corresponding to the phase-separated PBS-rich and
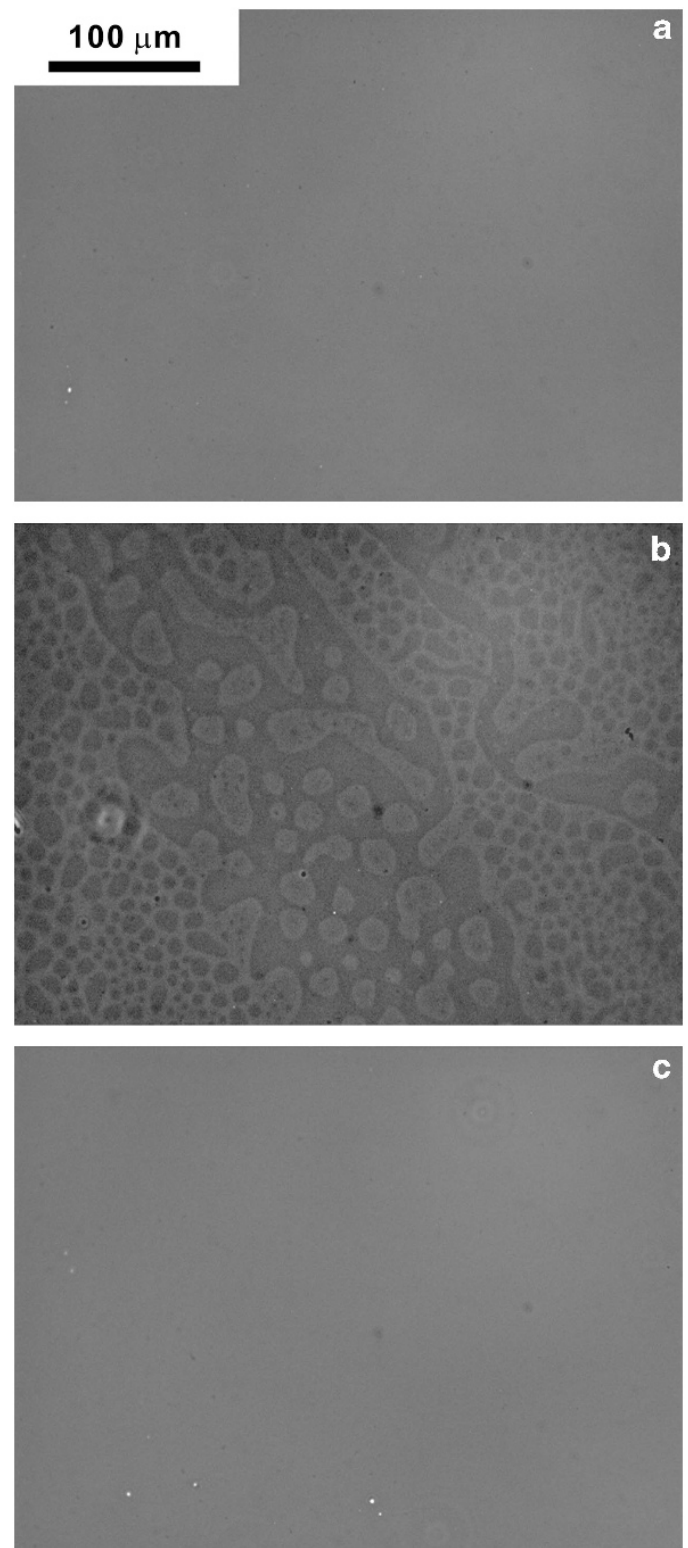

Figure 1 Phase contrast micrographs of the melt of the 4/6 PBS/PES blend in the homogeneous state at $180^{\circ} \mathrm{C}$ (a), in the phase-separated state at $130{ }^{\circ} \mathrm{C}(\mathrm{b})$ and of the homogeneous melt of the blend $4 / 6-60 \mathrm{~m}$ at $130{ }^{\circ} \mathrm{C}$ (c)

PES-rich phases in their amorphous states. However, the 4/6-60m blend, which was confirmed to be miscible by optical microscopy, showed a single $T_{\mathrm{g}}$. It should be noted that it is difficult to evaluate the miscibility by DSC if the two phase-separated melts have $T_{\mathrm{g}} \mathrm{s}$ close to each other. For instance, $4 / 6-30 \mathrm{~m}$ did not show two $T_{\mathrm{g}} \mathrm{s}$ on the thermogram despite its obvious phase separation in Figure 2. The single $T_{\mathrm{g}} \mathrm{s}$ for the annealed blends indicate enhancements in miscibility, which should be ascribed to the shorter block lengths induced by the transesterification.

The composition dependence of the $T_{\mathrm{g}}$ in the PBS/PES blends is shown in Figure 5. The PBS/PES blends without annealing and those annealed for $60 \mathrm{~min}$ showed two $T_{\mathrm{g}} \mathrm{s}$ and single $T_{\mathrm{g}} \mathrm{s}$, respectively. The $T_{\mathrm{g}} \mathrm{s}$ of the annealed blends increased with the weight fraction of PES. The $T_{\mathrm{g}}$ was almost insensitive to the annealing time. This most likely indicates that the $T_{\mathrm{g}}$ depends only on the annealed blend composition. 


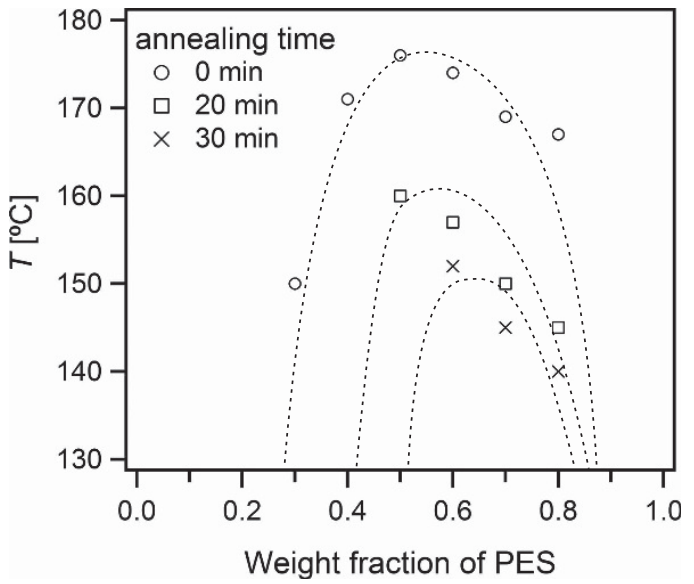

Figure 2 Phase separation temperatures of the PBS/PES blends annealed for $0-60 \mathrm{~min}$. The blends annealed for $60 \mathrm{~min}$ did not show phase separation above $130{ }^{\circ} \mathrm{C}$. The broken lines are to guide the eye.
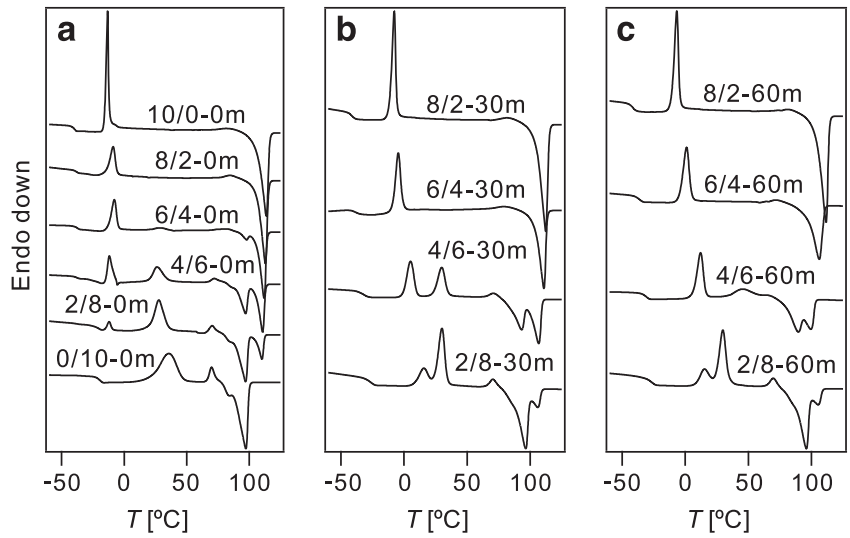

Figure 3 DSC heating curves of the PBS/PES blends annealed for 0 min (a), $30 \mathrm{~min}(\mathbf{b})$ and $60 \mathrm{~min}(\mathbf{c})$.

\section{Melting temperatures}

Figure 6 shows the $T_{\mathrm{m}} \mathrm{s}$ for the PBS/PES blends determined from the endothermic peaks in the DSC thermograms in Figure 3. The 6/4, 4/6 and 2/8 unannealed PBS/PES blends showed two $T_{\mathrm{m}} \mathrm{s}$ corresponding to the PBS and to the PES crystals. These $T_{\mathrm{m}} \mathrm{s}$ decreased gradually with increasing annealing time. However, the miscible blends annealed for $60 \mathrm{~min}$ still had $T_{\mathrm{m}} \mathrm{s}$ for PBS or PES higher than $90^{\circ} \mathrm{C}$ for all of the weight ratios.

These results indicate that the block copolymers formed in the melt-annealing process still contain longer PBS and PES sequences for crystallization than those obtained by condensation copolymerization ${ }^{45-48}$ when the annealing time is at least $60 \mathrm{~min}$. The $T_{\mathrm{m}}$ of the poly(butylene succinate-co-ethylene succinate) random copolymer was only $50{ }^{\circ} \mathrm{C}$ when the ethylene succinate unit was 53 mol\%. ${ }^{45}$

\section{Transesterification}

The molecular weight distribution before and after melt-annealing was evaluated based on the GPC profile in Figure 7, which shows chromatograms for PBS, PES and the PBS/PES blends annealed for 0-60 min. The chromatogram of the unannealed blend showed bimodal peaks representing PBS and PES. The annealed blends exhibited a broad unimodal peak between the peaks for neat PBS

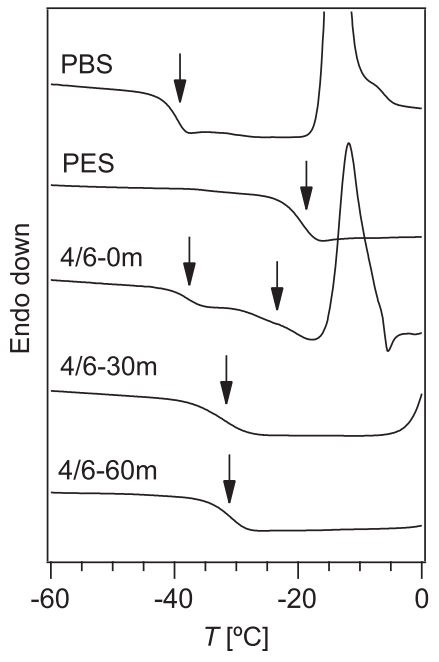

Figure 4 DSC thermograms of neat PBS, neat PES, and the 4/6 unannealed and annealed PBS/PES blends near the $T_{\mathrm{g}}$. The arrows denote the $T_{\mathrm{gS}}$.

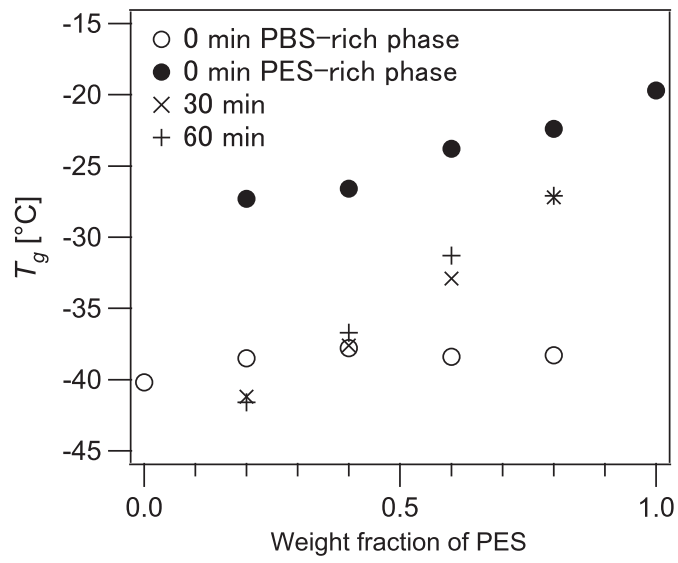

Figure 5 The weight fraction dependence of the $T_{\mathrm{g}} \mathrm{s}$ in the blends annealed for $0-60 \mathrm{~min}$.

and PES. This obviously indicates that the polymers efficiently reacted with each other during the annealing process under mild conditions (reaction temperature $\sim 150^{\circ} \mathrm{C}$ ). The transesterification reaction was generally carried out at high temperatures $\left(200-300{ }^{\circ} \mathrm{C}\right)$ with mechanical mixing of the melt and the addition of a $\mathrm{Ti}$ catalyst. ${ }^{21-23,28,29}$ Thus, this result can most likely be ascribed to the partial miscibility and low $T_{\mathrm{g}}$ s. The annealed blends also showed little decomposition because no significant signals from low-molecularweight components were observed in the GPC curve. Obtaining evidence for transesterification by ${ }^{1} \mathrm{H}$ NMR is very difficult because the repeating units of PBS and PES have similar chemical structures (see Supplementary Figures S1 and S2 in Supplementary Information).

The GPC results are analogous to what was reported by Otsuka et al., ${ }^{49}$ who demonstrated that a mixture of high- and lowmolecular-weight prepolymers with intermolecularly exchangeable covalent bonds on their main chains changed into intermediatemolecular-weight postpolymers after the interchange reaction. This is a characteristic tendency of main-chain exchange between reactive polymers. Moreover, a lowered $T_{\mathrm{m}}$ and the enhancement in miscibility have been reported after transesterification. ${ }^{26}$ On the basis of these 


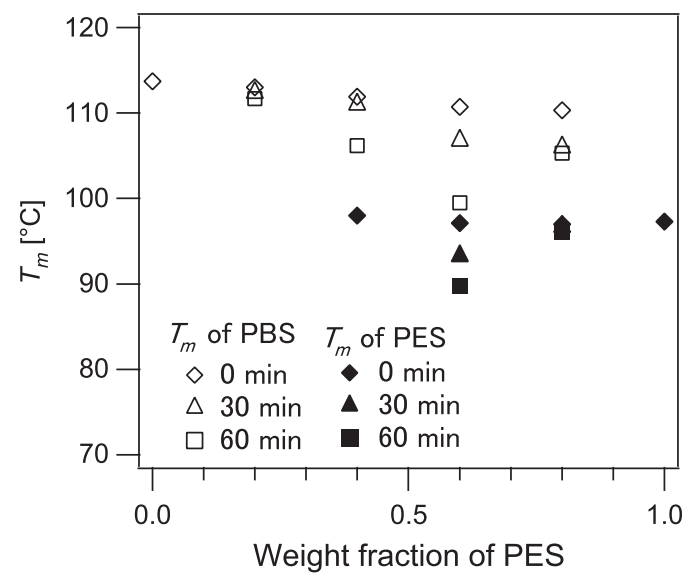

Figure 6 The weight fraction dependence of the $T_{\mathrm{m}} \mathrm{S}$ of PBS and PES in the blends annealed for $0-60 \mathrm{~min}$.

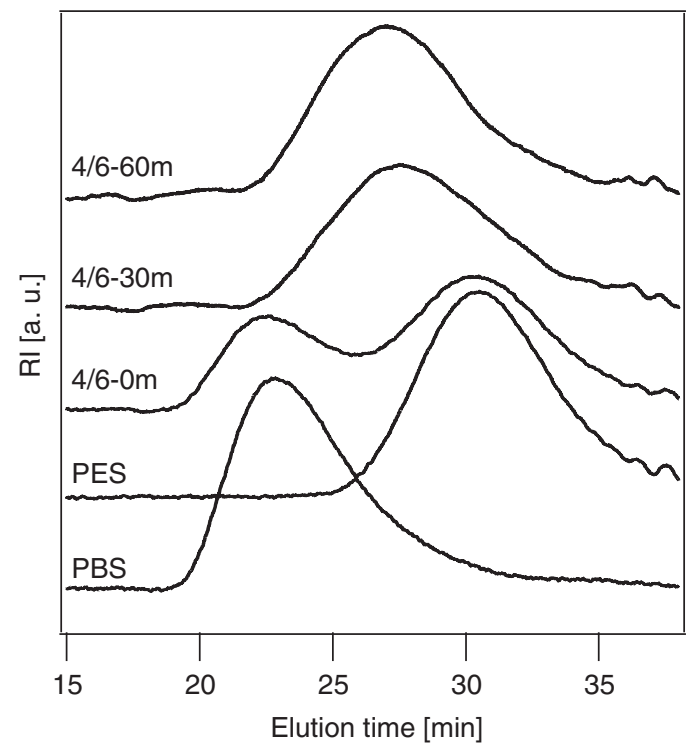

Figure 7 GPC profiles for neat PBS, neat PES and the 4/6 unannealed and annealed PBS/PES blends.

previous studies, transesterification between PBS and PES should occur when the PBS/PES blends are annealed.

\section{Crystallization of the unannealed PBS/PES blends}

The 6/4, 4/6 and 2/8 unannealed PBS/PES blends showed phase separation at temperatures near the $T_{\mathrm{m}}$ of PBS. Both PBS and PES crystallized upon cooling. However, only PBS crystallized from a homogeneous melt of the $8 / 2$ blend most likely because of the small PES content.

A typical example of isothermal crystallization of an unannealed blend is shown in Figure 8. When the heterogeneous melt of the 4/6 $\mathrm{PBS} / \mathrm{PES}$ blend was quenched to $80{ }^{\circ} \mathrm{C}$, PBS spherulites nucleated and grew, as shown in Figures $8 \mathrm{a}$ and $\mathrm{b}$. The brighter parts of the spherulites correspond to the PBS-rich phase. PBS appeared to also crystallize in the PES-rich phase (the darker part) because the spherulites continued to grow across the phase boundary. The growth front of the PBS spherulites was spherical in shape. This indicates that the spherulitic growth rate is constant despite the large difference in the PBS concentration between the PBS-rich and the PES-rich phases. It is noted that the spherulitic growth rate usually depends on the composition. The present result is most likely ascribed to the formation of a layered structure in which a thin PBS-rich layer exists between the PES-rich phase and the glass substrate. PBS crystallized in the thin PBS-rich layer in the darker part of the spherulite in Figures $8 \mathrm{a}$ and $\mathrm{b}$.

PES spherulites also nucleated in the PES-rich phase after cooling to $50{ }^{\circ} \mathrm{C}$ (Figures $8 \mathrm{a}$ and $\mathrm{d}$ ). The additive birefringence (blue color) indicated by the arrows in Figure 8b, which is in the second and the forth quadrants of the PBS spherulite in the PES-rich phase, changed after the growth of the PES spherulites (Figure 8d). This result indicates the overlap of the PBS and PES spherulites and the existence of the layered structure.

Immiscible binary blends do not generally form such layers if the characteristic length of the phase-separated structure is much larger than the film thickness because the surface free energy increases in the layered structures. The PBS-PES block copolymers between the PBSrich and the PES-rich phases might stabilize the layered structure in the immiscible PBS/PES melt. Though the blend was not intentionally annealed, a small amount of block copolymers may have formed through transesterification when the sample was melted for a short time. These copolymers usually exist at the interface between two phases in an immiscible blend.

\section{Crystallization of the melt-annealed PBS/PES blends}

The crystallization behavior of the annealed blends was different from that of the unannealed blends because of the miscibility of the blends. Both PBS and PES crystallized in the 6/4-60 $\mathrm{m}, 4 / 6-60 \mathrm{~m}$ and $2 / 8-$ $60 \mathrm{~m}$ blends, whereas only PBS crystallized in the $8 / 2-60 \mathrm{~m}$ because of the low PES content.

The PBS and PES in the 6/4-60 $\mathrm{m}, 4 / 6-60 \mathrm{~m}$ and 2/8-60 $\mathrm{m}$ blends crystallized successively. Figure 9 shows the crystallization process for the $4 / 6-60 \mathrm{~m}$ blend. When the homogeneous melt was cooled to $50{ }^{\circ} \mathrm{C}$, the PBS spherulites nucleated first and filled the whole volume without phase separation (Figures $9 \mathrm{a}$ and b). Shortly after the completion of the crystallization of PBS, PES spherulites nucleated and grew slowly inside the PBS spherulites, as observed in the brighter spherulitic parts shown in Figure 9c.

When the PES spherulites grew inside the PBS spherulites, the PBS spherulites became brighter, while maintaining their original morphology (Figures $9 \mathrm{c}$ and $\mathrm{d}$ ). This result indicates the formation of interpenetrating spherulites, which have been described in previous papers. ${ }^{9,10,16,17}$ This morphology is different from that of the unannealed blends, which contain layered structures (Figures $8 \mathrm{c}$ and $\mathrm{d}$ ).

However, the two components in 2/8-60 $\mathrm{m}$ showed simultaneous crystallization from the melt. Figure 10 shows the crystallization process of the $2 / 8-60 \mathrm{~m}$ blend quenched to $60^{\circ} \mathrm{C}$ from the homogenous melt. Many PBS crystals nucleated, and a PES spherulite appeared at the center. The spherulitic growth rate of the PES was faster than that of the PBS. After the PBS and PES spherulites impinged on each other, the PBS spherulites became brighter, while maintaining their morphology, indicating that the PES crystallized inside the PBS spherulites (Figure 10b).

Simultaneous crystallization usually occurs when the $T_{\mathrm{m}} \mathrm{s}$ of the two constituents are close to each other., ${ }^{5,9}$ The present result was most likely caused by the large melting-point depression of PBS due to the small content of PBS in the 2/8-60 m blend.

The distances of spherulitic growth in the melt and inside the spherulites of the other component are shown in Figure 11. After the growth fronts of the PBS and PES spherulites contacted each other, 

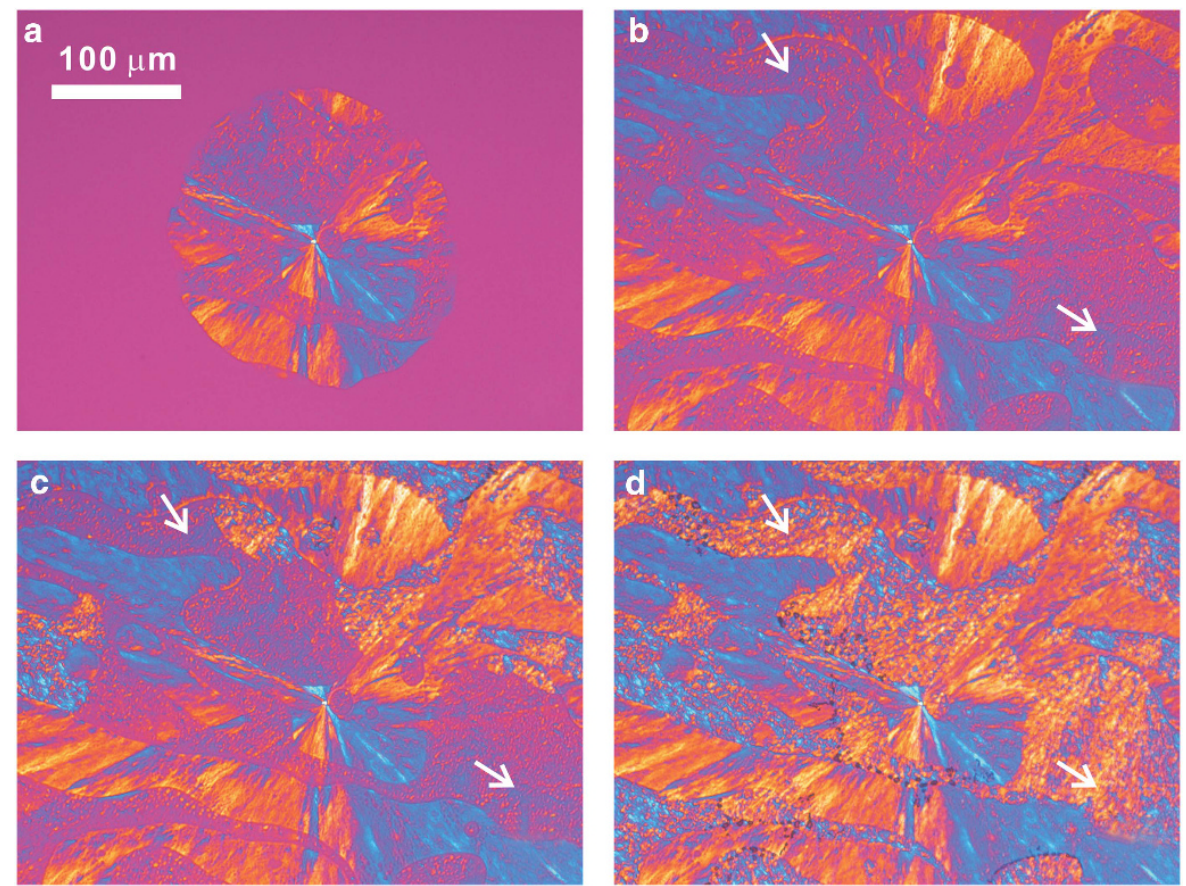

Figure 8 Polarized optical micrographs of the crystallization process for the 4/6 PBS/PES blend. (a) Nucleation and growth of a PBS spherulite in the phase-separated melt at $80^{\circ} \mathrm{C}$. The brighter parts of the spherulite are in the PBS-rich phase. (b) Completion of the spherulitic growth of the PBS. The arrows indicate additive birefringence of the PBS spherulite in the PES-rich phase. (c) Nucleation and growth of a PES spherulite inside the PES-rich phase after quenching at $50^{\circ} \mathrm{C}$. (d) Completion of the spherulitic growth of the PES.
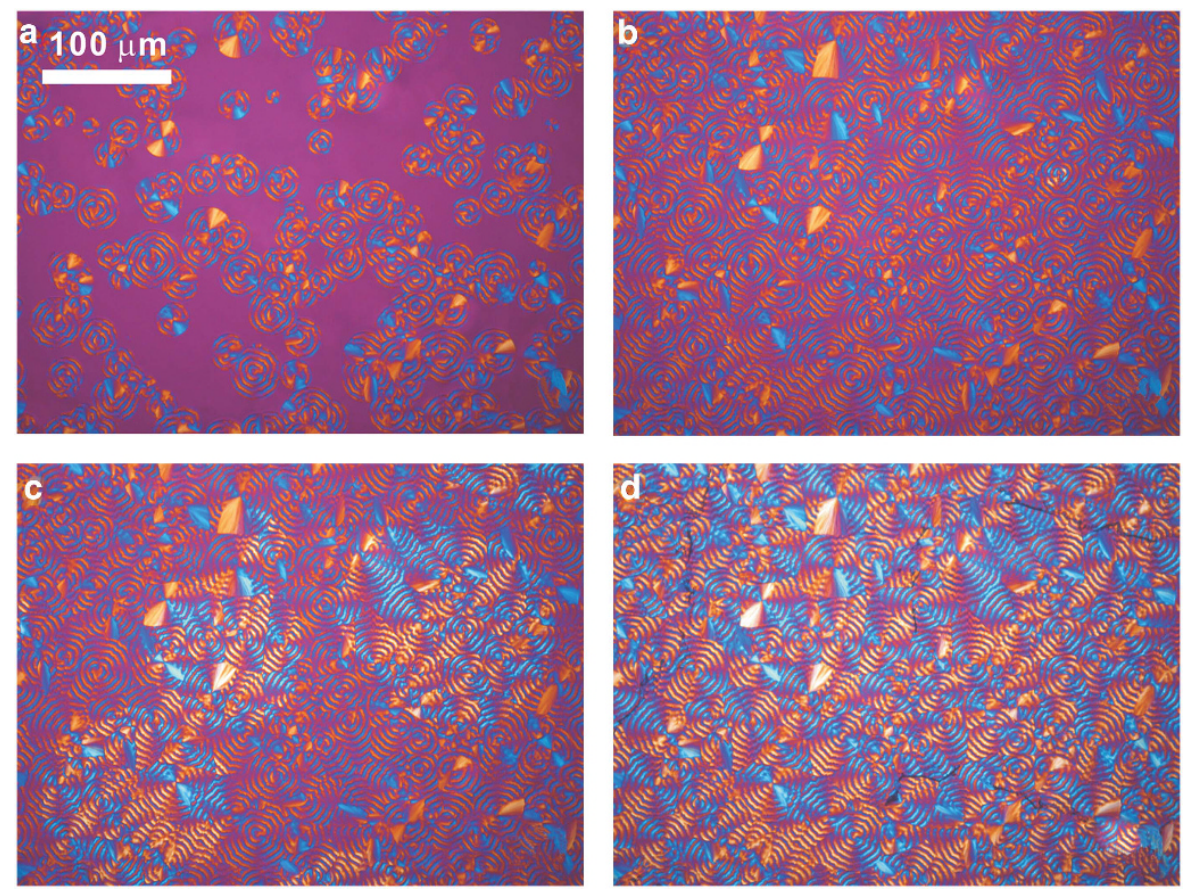

Figure 9 Polarized optical micrographs of the successive crystallization processes in the $4 / 6-60 \mathrm{~m}$ blend quenched at $50^{\circ} \mathrm{C}$. (a) Nucleation of the PBS spherulites from the homogeneous melt. (b) Completion of the spherulitic growth of the PBS. (c) Nucleation and growth of the PES spherulites inside the PBS spherulites. (d) Completion of the spherulitic growth of the PES.

the spherulitic growth rate of the PES changed slightly, whereas the growth of the PBS spherulite almost stopped. This indicates that the growth of the PBS spherulite was significantly suppressed by the existence of the PES spherulite.
It has been reported that for interpenetrating spherulites, the spherulitic growth rate of one component inside the spherulites of the other component is often different from that in the melt. ${ }^{11,16}$ The asymmetric composition of the $2 / 8-60 \mathrm{~m}$ blend resulted in a large 

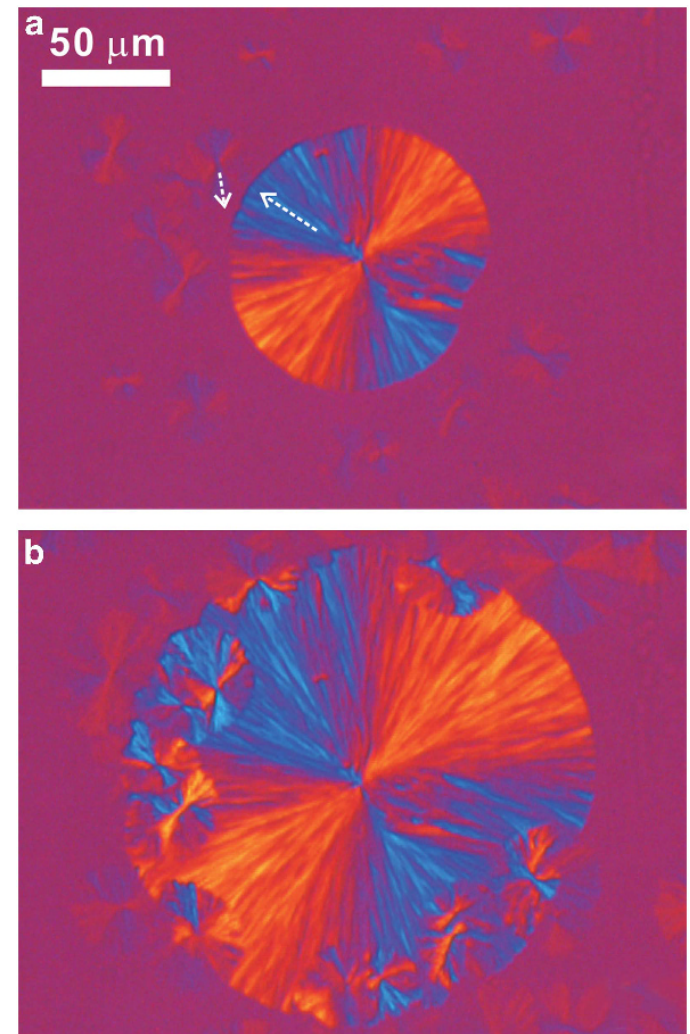

Figure 10 Polarized optical micrographs of the simultaneous spherulitic growth of PBS and PES in the $2 / 8-60 \mathrm{~m}$ blend at $60^{\circ} \mathrm{C}$. (a) Nucleation and spherulitic growth of PBS and PES in the homogeneous melt. The brighter and the darker spherulites correspond to PES and PBS, respectively. The spherulitic growth distances in the directions of the arrows are plotted against time in Figure 11. (b) Penetrating growth of the PES spherulites into the PBS spherulites.

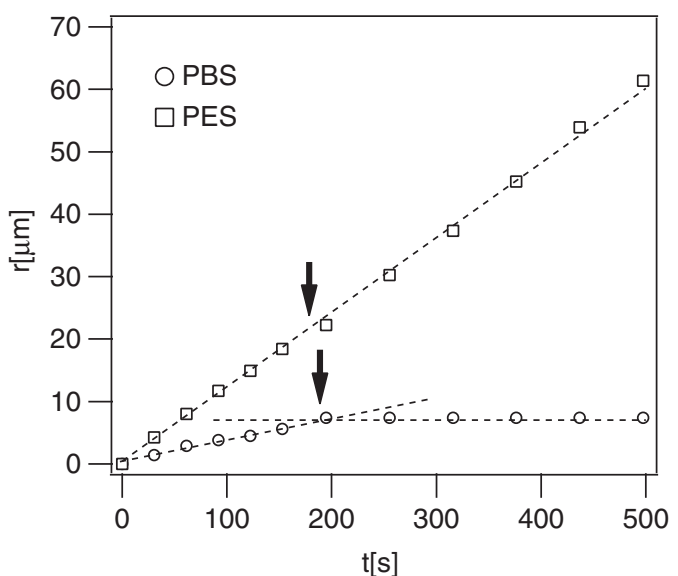

Figure 11 Time dependence of the spherulitic growth distance, $r$, in the directions of the dashed arrows in Figure 10. The arrows in this figure denote the time at which the spherulitic growth front reaches the spherulites of the other component.

amount of amorphous PES in the PBS spherulites, which enabled PES to crystallize inside. However, the small PBS content in the amorphous regions in the PES spherulites most likely suppressed the crystallization of PBS in the PES spherulites.

\section{CONCLUSIONS}

The melt annealing of immiscible PBS/PES blends resulted in miscible crystalline/crystalline binary blends. The GPC and DSC studies implied that annealing caused the formation of copolyesters through transesterification between the PBS and the PES, resulting in an enhancement in miscibility. The $T_{\mathrm{m}} \mathrm{s}$ of PBS and PES in the annealed blends were higher than those of the random copolymers obtained by typical polycondensation. ${ }^{45}$ The unannealed blends showed phase separation near the $T_{\mathrm{m}}$ of PBS. Separate crystallization of PBS and PES was observed in the PBS- and PES-rich phases, respectively. A thin PBS-rich layer formed between the PES-rich phase and the glass substrate. However, the annealed blends showed two types of crystallization: successive crystallization, where the PBS crystallized from the melt and then PES spherulites nucleated from the PBS spherulites and simultaneous nucleation of both substances, where the PES spherulites nucleated from the melt and continued to grow inside the PBS spherulites after the growth front of the PBS and PES spherulites impinged on each other.

\section{ACKNOWLEDGEMENTS}

The authors thank Mr Masaki Nakokohji for his help. This work was partially supported by a Grant-in-Aid for Scientific Research (C) from the Japan Society for the Promotion of Science (22550201).

1 Amass, W., Amass, A. \& Tighe, B. A review of biodegradable polymers: uses, current developments in the synthesis and characterization of biodegradable polyesters, blends of biodegradable polymers and recent advances in biodegradation studies. Polym. Int. 47, 89-144 (1998).

2 Yu, L., Dean, K. \& Li, L. Polymer blends and composites from renewable resources. Prog. Polym. Sci. 31, 576-602 (2006).

3 Papageorgiou, G. Z. \& Bikiaris, D. N. Biodegradable poly(alkylene succinate) blends: Thermal behavior and miscibility study. J. Polym. Sci. Part B: Polym. Phys. 44 584-597 (2006)

4 He, Y. S., Zeng, J. B., Li, S. L. \& Wang, Y. Z. Crystallization behavior of partially miscible biodegradable poly(butylene succinate)/poly(ethylene succinate) blends. Thermochim. Acta 529, 80-86 (2012).

5 Blumm, E. \& Owen, A. J. Miscibility, crystallization and melting of poly(3-Hydroxybutyrate)/poly(L-Lactide) blends. Polymer 36, 4077-4081 (1995).

6 Qiu, Z. B., Fujinami, S., Komura, M., Nakajima, K., Ikehara, T. \& Nishi, T. Structure and properties of biodegradable polymer-based blends. Macromol. Symp. 216, 255-263 (2004).

7 Lee, J. C., Tazawa, H., Ikehara, T. \& Nishi, T. Crystallization kinetics and morphology in miscible blends of two crystalline polymers. Polym. J. 30, 780-789 (1998).

8 Ikehara, T. \& Nishi, T. Interpenetrated spherulites of poly(butylene succinate) poly(vinylidene chloride-co-vinyl chloride) blends. An optical microscopic study. Polym. J. 32, 683-687 (2000).

9 Ikehara, T., Kimura, H. \& Qiu, Z. B. Penetrating spherulitic growth in poly(butylene adipate-co-butylene succinate)/poly(ethylene oxide) blends. Macromolecules $\mathbf{3 8}$ 5104-5108 (2005).

10 Ikehara, T., Kurihara, H. \& Kataoka, T. Effect of poly(butylene succinate) crystals on spherulitic growth of poly(ethylene oxide) in binary blends of the two substances. J. Polym. Sci. Part B: Polym. Phys. 47, 539-547 (2009).

11 Qiu, Z. B., Ikehara, T. \& Nishi, T. Unique morphology of poly(ethylene succinate)/ poly(ethylene oxide) blends. Macromolecules 35, 8251-8254 (2002).

12 Ikehara, T., Kurihara, H., Qiu, Z. B. \& Nishi, T. Study of spherulitic structures by analyzing the spherulitic growth rate of the other component in binary crystalline polymer blends. Macromolecules 40, 8726-8730 (2007)

13 Qiu, Z. B., Yan, C. Z., Lu, J. M., Yang, W. T., Ikehara, T. \& Nishi, T. Various crystalline morphology of poly(butylene succinate-co-butylene adipate) in its miscible blends with poly(vinylidene fluoride). J. Phys. Chem. B 111, 2783-2789 (2007).

14 Qiu, Z. B., Komura, M., Ikehara, T. \& Nishi, T. Miscibility and crystallization behavior of biodegradable blends of two aliphatic polyesters. Poly(butylene succinate) and poly (E-caprolactone). Polymer 44, 7749-7756 (2003).

15 Qiu, Z. B., Ikehara, T. \& Nishi, T. Miscibility and crystallization in crystalline/crystalline blends of poly(butylene succinate)/poly(ethylene oxide). Polymer 44, 2799-2806 (2003).

16 Ikehara, T., Kurihara, H. \& Kataoka, T. Spherulitic growth in block copolymers and blends of miscible crystalline polymers. J. Polym. Sci. Part B: Polym. Phys. 50, 563-571 (2012).

17 Ikehara, T., Kurihara, H., Qiu, Z. \& Nishi, T. Study of spherulitic structures by analyzing the spherulitic growth rate of the other component in binary crystalline polymer blends. Macromolecules 40, 8726-8730 (2007). 
18 Utracki, L. A. Compatibilization of polymer blends. Can. J. Chem. Eng. 80, 1008-1016 (2002).

19 Shi, Y. \& Jabarin, S. A. Transesterification reaction kinetics of poly(ethylene terephthalate/poly(ethylene 2,6-naphthalate) blends. J. Appl. Polym. Sci. 80, 2422-2436 (2001).

20 Shi, Y. \& Jabarin, S. A. Class-transition and melting behavior of poly(ethylene terephthalate)/poly(ethylene 2,6-naphthalate) blends. J. Appl. Polym. Sci. 81, 11-22 (2001).

21 Kim, S. W., Lim, J. C., Kim, D. J. \& Seo, K. H. Synthesis and characteristics of biodegradable copolyesters from the transesterification of poly(butylene adipate-cosuccinate) and poly(ethylene terephthalate). J. Appl. Polym. Sci. 92, 3266-3274 (2004).

22 Montaudo, G., Puglisi, C. \& Samperi, F. Mechanism of exchange in PBT/PC and PET/ PC blends. Composition of the copolymer formed in the melt mixing process. Macromolecules 31, 650-661 (1998).

23 Kong, Y. \& Hay, J. N. The effect of annealing on the crystallization of poly(ethylene terephthalate)/polycarbonate blends. J. Polym. Sci. Part B: Polym. Phys. 42, 2129-2136 (2004).

24 Raquez, J. M., Narayan, R. \& Dubois, P. Recent advances in reactive extrusion processing of biodegradable polymer-based compositions. Macromol. Mater. Eng. 293, 447-470 (2008).

25 Lin, S., Guo, W. N., Chen, C. Y., Ma, J. L. \& Wang, B. B. Mechanical properties and morphology of biodegradable poly(lactic acid)/poly(butylene adipate-co-terephthalate) blends compatibilized by transesterification. Mater. Design 36, 604-608 (2012).

26 Maeda, T., Otsuka, H. \& Takahara, A. Dynamic covalent polymers: reorganizable polymers with dynamic covalent bonds. Prog. Polym. Sci. 34, 581-604 (2009).

27 Porter, R. S. \& Wang, L. H. Compatibility and transesterification in binary polymer blends. Polymer 33, 2019-2030 (1992).

28 Soccio, M., Lotti, N., Gigli, M., Finelli, L., Gazzano, M. \& Munari, A. Reactive blending of poly(butylene succinate) and poly(triethylene succinate): characterization of the copolymers obtained. Polym. Int. 61, 1163-1169 (2012).

29 El Shafee, E., Zaki, M. \& Saad, G. R. Annealing of poly(trimethylene terephthalate)/ polycarbonate blends. J. Polym. Res. 16, 317-327 (2009).

30 Kugler, J., Gilmer, J. W., Wiswe, D., Zachmann, H. G., Hahn, K. \& Fischer, E. W. Study of transesterification in poly(ethylene terephthalate) by small-angle neutron scattering. Macromolecules 20, 1116-1119 (1987).

31 Yoon, H., Feng, Y., Qiu, Y. \& Han, C. C. Structural stabilization of phase separating $\mathrm{PC} /$ polyester blends through interfacial modification by transesterification reaction. J. Polym. Sci. Part B: Polym. Phys. 32, 1485-1492 (1994).

$32 \mathrm{Xu}$, J. \& Guo, B. H. Poly(butylene succinate) and its copolymers: research, development and industrialization. Biotechnol. J. 5, 1149-1163 (2010).

33 Lee, C. W., Kimura, Y. \& Chung, J. D. Mechanism of enzymatic degradation of poly(butylene succinate). Macromol. Res. 16, 651-658 (2008).

34 Chrissafis, K., Paraskevopoulos, K. M. \& Bikiaris, D. N. Thermal degradation mechanism of poly(ethylene succinate) and poly(butylene succinate): comparative study. Thermochim. Acta 435, 142-150 (2005).
35 Qiu, Z. B., Fujinami, S., Komura, M., Nakajima, K., Ikehara, T. \& Nishi, T. Nonisothermal crystallization kinetics of poly(butylene succinate) and poly(ethylene succinate). Polym. J. 36, 642-646 (2004).

36 Qiu, Z. B., Komura, M., Ikehara, T. \& Nishi, T. DNC and TMDSC study of melting behaviour of poly(butylene succinate) and poly(ethylene succinate). Polymer 44, 7781-7785 (2003).

37 Phua, Y. J., Chow, W. A. \& Ishad, Z. A. M. Mechanical properties and structure development in poly(butylene succinate)/organo-montmorillonite nanocomposites under uniaxial cold rolling. Express. Polym. Lett. 5, 93-103 (2011).

$38 \mathrm{Li}$, H. Y., Chang, J., Cao, A. M. \& Wang, J. Y. In vitro evaluation of biodegradable poly(butylene succinate) as a novel biomaterial. Macromol. Biosci. 5, 433-440 (2005).

39 Tachibana, Y., Masuda, T., Funabashi, M. \& Kunioka, M. Chemical synthesis of fully biomass-based poly(butylene succinate) from inedible-biomass-based furfural and evaluation of its biomass carbon ratio. Biomacromolecules 11, 2760-2765 (2010).

40 Qiu, Z. B., Ikehara, T \& Nishi, T. Crystallization behaviour of biodegradable poly(ethylene succinate) from the amorphous state. Polymer 44, 5429-5437 (2003).

41 Hoang, K. C., Tseng, M. \& Shu, W. J. Degradation of polyethylene succinate (PES) by a new thermophilic Microbispora strain. Biodegradation 18, 333-342 (2007).

42 Ikehara, T., Kimura, H. \& Kataoka, T. Miscibility enhancement and formation of interpenetrating spherulites in ternary blends of crystalline polymers. J. Polym. Sci. Part B: Polym. Phys. 48, 706-711 (2010).

43 Zeng, J. B., Zhu, Q. Y., Lu, X., He, Y. S. \& Wang, Y. Z. From miscible to partially miscible biodegradable double crystalline poly (ethylene succinate)- $b$-poly(butylene succinate) multiblock copolymers. Polym. Chem-UK 3, 399-408 (2012).

44 Zhu, Q. Y., He, Y. S., Zeng, J. B., Huang, Q. \& Wang, Y. Z. Synthesis and characterization of a novel multiblock copolyester containing poly(ethylene succinate) and poly(butylene succinate). Mater. Chem. Phys. 130, 943-949 (2011).

45 Mochizuki, M. Mukai, K. Yamada, K., Ichise, N., Murase, S. \& Iwaya, Y Structural effects upon enzymatic hydrolysis of poly(butylene succinate-co-ethylene succinate)s. Macromolecules 30, 7403-7407 (1997).

46 Chen, C. H., Lu, H. Y., Chen, M., Peng, J. S., Tsai, C. J. \& Yang, C. S. Synthesis and characterization of poly(ethylene succinate) and its copolyesters containing minor amounts of butylene succinate. J. Appl. Polym. Sci. 111, 1433-1439 (2009).

47 Cao, A., Okamura, T., Nakayama, K., Inoue, Y. \& Masuda, T. Studies on syntheses and physical properties of biodegradable aliphatic poly(butylene succinate-co-ethylene succinate)s and poly(butylene succinate-co-diethylene glycol succinate)s. Polym. Degrad. Stabil. 78, 107-117 (2002).

48 Gan, Z., Abe, H. \& Doi, Y. Crystallization, melting, and enzymatic degradation of biodegradable poly(butylene succinate-co-14 mol \% ethylene succinate) copolyester. Biomacromolecules 2, 313-321 (2001).

49 Otsuka, H., Aotani, K., Higaki, Y., Amamoto, Y. \& Takahara, A. Thermal reorganization and molecular weight control of dynamic covalent polymers containing alkoxyamines in their main chains. Macromolecules 40, 1429-1434 (2007).

Supplementary Information accompanies the paper on Polymer Journal website (http://www.nature.com/pj) 